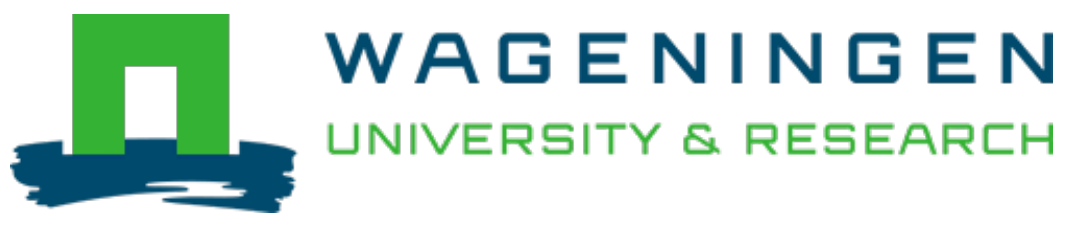

\title{
A Fast GC-MS based method for efficacy assessment of natural anti-oxidants for inhibiting lipid oxidation
}

\author{
Journal of AOAC International \\ Janssen, H.G.; Gah, C.; Steenbergen, H.; Rosing, E.; Spraul, M. \\ https://doi.org/10.1093/jaoacint/qsaa088
}

This publication is made publicly available in the institutional repository of Wageningen University and Research, under the terms of article $25 \mathrm{fa}$ of the Dutch Copyright Act, also known as the Amendment Taverne. This has been done with explicit consent by the author.

Article 25 fa states that the author of a short scientific work funded either wholly or partially by Dutch public funds is entitled to make that work publicly available for no consideration following a reasonable period of time after the work was first published, provided that clear reference is made to the source of the first publication of the work.

This publication is distributed under The Association of Universities in the Netherlands (VSNU) 'Article $25 \mathrm{fa}$ implementation' project. In this project research outputs of researchers employed by Dutch Universities that comply with the legal requirements of Article $25 \mathrm{fa}$ of the Dutch Copyright Act are distributed online and free of cost or other barriers in institutional repositories. Research outputs are distributed six months after their first online publication in the original published version and with proper attribution to the source of the original publication.

You are permitted to download and use the publication for personal purposes. All rights remain with the author(s) and / or copyright owner(s) of this work. Any use of the publication or parts of it other than authorised under article $25 \mathrm{fa}$ of the Dutch Copyright act is prohibited. Wageningen University \& Research and the author(s) of this publication shall not be held responsible or liable for any damages resulting from your (re)use of this publication.

For questions regarding the public availability of this publication please contact openscience.library@wur.nl 


\title{
A Fast GC-MS-Based Method for Efficacy Assessment of Natural Anti-Oxidants for Inhibiting Lipid Oxidation
}

\author{
Hans-Gerd Janssen $\odot{ }^{1,2}$ Carmen Gah, ${ }^{3}$ Herrald Steenbergen, ${ }^{1}$ Ed Rosing, ${ }^{1}$ \\ and Martin Spraul ${ }^{3}$ \\ ${ }^{1}$ Unilever Foods Innovation Centre, Science and Technology, Bronland 14, $6708 \mathrm{WH}$, Wageningen, The \\ Netherlands, ${ }^{2}$ Wageningen University, Agrotechnology and Food Sciences Group, Laboratory of Organic \\ Chemistry, Stippeneng 4, 6708 WE, Wageningen, The Netherlands, ${ }^{3}$ Unilever R\&D Savoury, Knorrstrasse 1, \\ D-74074 Heilbronn, Germany
}

Corresponding author's e-mail: hans-gerd.janssen@unilever.com

\begin{abstract}
Background: For health reasons it is preferred to prepare food products with edible fats and oils that are high in unsaturated fatty acids. Unfortunately, these unsaturated acids are susceptible to lipid oxidation and the addition of natural antioxidants, e.g., rosemary extracts, etc. is needed.

Objective: To assess the efficacy of natural oxidation inhibition strategies, fast, yet realistic, and objective methods are needed to study oxidation inhibition.

Methods: A model system consisting of salt and sunflower oil is proposed as a model for dry soups and sauces. Hexanal formation is studied using fast GC-MS as a quantitative indicator for lipid oxidation.

Results: A fast GC-MS method using a short, 6-m $150 \mu \mathrm{m}$ inner-diameter column was developed that allowed elution of hexanal within approximately $20 \mathrm{~s}$, with a total run time of $2 \mathrm{~min}$. The GC method has quantification limits below $1 \mathrm{ppm}$ and is hence much more sensitive than the human nose.

Conclusions: The new accelerated method with hexanal read-out was successfully applied in a study to identify spices and herbs mixtures that can act as natural inhibitors of lipid oxidation. The fast GC-MS method is extremely stable and allowed the analysis of thousands of samples with very little maintenance.

Highlights: With the right mixture of spices and herbs, lipid oxidation can be delayed more than 100 times as compared to non-stabilized systems.
\end{abstract}

Oxidation of lipids in food products produces a range of volatile off-flavor compounds resulting in the typical rancid off-smell of aging foods. The presence of this rancid smell is a clear indicator for the loss of quality of the food. Understanding lipid oxidation and developing methods for preventing, or at least delaying, it has received a great deal of attention in foods research. Extensive research has been done on antioxidants, compounds that can be added to the food matrix in small quantities to prevent or retard lipid oxidation. This has resulted in a whole range of synthetic antioxidants and, more recently, the use of several natural antioxidants, including spices and herbs such as rosemary, thyme, and coriander $(1,2)$.

Oxidation of lipids in food products can proceed according to three main pathways, chemical-, light-induced, and enzymatic oxidation (3). Chemical oxidation is the most frequently seen route. It follows a radical mechanism and will in principle occur in all situations where oxygen is present. Antioxidants delay chemical oxidation by intervening in the radical mechanism. Measuring radical scavenging activity of mixtures is hence a first option to test antioxidant efficacy. However, as stated by 
Becker et al., assessment of radical scavenging activity is far from reality (4). It can hence only be a first step in determining antioxidant capacity and should be followed by more realistic storage tests. Since oxidative changes in model systems are often too slow to be detected within an acceptable time frame, acceleration is needed, for example by working at elevated temperatures or increased oxygen levels.

In addition to the conditions for the accelerated aging studies, the actual read-out of the experiment also should be defined. Sensory assessment of the aging model sample after different exposure times would be the best reflection of consumer experience. Unfortunately, this route is neither fully objective nor quantitative, making it difficult to obtain comparable data over longer time periods. Head-space analysis, for example by gas chromatography, is closest to sensory assessment. Ideally, the head-space method targets the newly formed compounds that are responsible for the off-flavor of the aged foods. For many widely used vegetable oils, hexanal is one of the key off-flavor compounds formed upon oxidation (5); it is hence a good oxidation product to monitor during the accelerated aging.

As regards the effects of antioxidants in radical-based oxidation reactions, two classes of antioxidants have traditionally been distinguished: primary or chain braking antioxidants and secondary or preventive antioxidants (6). Spices and herbs contain a wide range of compounds that might act according to both these mechanisms. Moreover, so-called redox cycling can occur where antioxidants that are consumed are regenerated by the action of other compounds present (7). As a consequence of this, and even more so because there are many spices and herbs, the development of an optimized antioxidant mix based on spices and herbs will require a large number of model experiments and hence a very large number of head-space GC hexanal measurements. The GC method that is employed should hence be very fast.

In this contribution we describe the development of a fast and simple accelerated model experiment for studying the antioxidant effects of spices and herbs mixtures. The key element in the experiment is a fast GC-MS method that allows rapid quantification of hexanal levels in a model system containing aging sunflower oil. Hexanal formation over time is monitored in a system with and without added antioxidant mixes. Injection conditions and detector settings of the GC-MS system are optimized to allow sensitive hexanal detection. The detection limit of the method is established and a method to express antioxidant activity in a quantitative manner is proposed. Finally, the method is applied to evaluate the efficacy of several spices and herbs mixtures and formats.

\section{Experimental}

\section{Instrumentation}

All GC-MS experiments were performed on an Agilent $7890 \mathrm{~A}$ GC-MS system (Agilent, Amstelveen, the Netherlands) equipped with a Gerstel MPS-XL autosampler (Gerstel, Mülheim a/d Ruhr, Germany) capable of head-space injections and containing a heated $2.5 \mathrm{~mL}$ head-space syringe. Injection was performed in the split mode with a split ratio of $25: 1$. The quartz liner of the injector had an internal diameter of $2 \mathrm{~mm}$. Injector temperature was $200^{\circ} \mathrm{C}$. The injection volume was $750 \mu \mathrm{L}$, both for the samples as well as for the calibration standards. The gaseous headspace samples were injected at a rate of $1000 \mu \mathrm{L} / \mathrm{s}$. The syringe temperature was kept at $50^{\circ} \mathrm{C}$. Carrier gas was Helium at a constant flow of $2.0 \mathrm{~mL} / \mathrm{min}$. The column was a short capillary column with an inner diameter of $150 \mu \mathrm{m}$, and a length of $6 \mathrm{~m}$ (Agilent, Middelburg, the Netherlands). It was coated with $0.3 \mu \mathrm{m}$ of a VF- $5 \mathrm{~ms}$ stationary phase. The oven temperature program started at $45^{\circ} \mathrm{C}(0 \mathrm{~min})$ and was programmed to $80^{\circ} \mathrm{C}$ $\left(0 \mathrm{~min}\right.$ ) at $80^{\circ} \mathrm{C} / \mathrm{min}$ followed by a ramp of $100^{\circ} \mathrm{C} / \mathrm{min}$ to $120^{\circ} \mathrm{C}$ $(0.16 \mathrm{~min})$. To allow the system to achieve these high heating rates reproducibly, the oven was equipped with a high-speed oven-insert pillow (Agilent). MS acquisition was performed in the SIM mode, monitoring the ions $\mathrm{m} / \mathrm{z} 56, \mathrm{~m} / \mathrm{z} 72$, and $\mathrm{m} / \mathrm{z} 82$. Ion 56 was used as quantifier ion, the other two as qualifiers.

\section{Accelerated Aging}

Sample aging was performed in a ATKF600.10.70 climate control cupboard (Airtest Solutions B.V., Malden, the Netherlands) at $30^{\circ} \mathrm{C}$. Aging was done in large, $250 \mathrm{~mL}$ glass jars with caps containing $3 \mathrm{~mm}$ PTFE coated silicone rubber inlays (VWR, Amsterdam, the Netherlands) for 11 weeks. In the final test protocol, we combined $100 \mathrm{~g}$ of fine, iodine-free kitchen salt with $1 \mathrm{~g}$ of sunflower oil both obtained from a local supermarket. The spices and herbs to test for their antioxidant properties were added to the bottle and the bottle was shaken for $5 \mathrm{~min}$ at $1500 \mathrm{rpm}$ in a VWR DVX-2500 multi-tube vortexer (VWR). Next, the bottles were stored in the climate cabinet at $30^{\circ} \mathrm{C}$ in the dark. Typical levels of the spices mixes added ranged from 0.05 to $0.2 \mathrm{~g}$. For the analysis of the headspace in the large jars after aging, the sample rack of the head space sampler was modified. In the modified rack only one sample jar could be put, at a welldefined, fixed position. Because the rack had only one position, samples had to be changed manually. To enable the recording of time curves, repeated sampling should be performed with intermediate aging in the climate cabinet. To avoid the loss of headspace during the analysis, all sample jars were covered with aluminum foil before closing them with the silicone-inlay caps. Upon sampling the sample syringe pierced the aluminum foil. After the sample had been taken, the hole created was closed by a piece of tape, the cap of the jar was put back, and the sample was put back into the climate chamber. Time curves obtained for the different bottles never showed real outliers, meaning the closing procedures works well. By turning the bottle slightly at every analysis point it could be avoided that the head-space sampler had to pierce through the tape. After approximately ten injections, an additional layer of aluminum foil was put over the first layer. For practical reasons, samples were allowed to reach room temperature (climate controlled at $21 \pm 1.0^{\circ} \mathrm{C}$ ) prior to head-space analysis. Initially, it was attempted to measure samples at $30^{\circ} \mathrm{C}$ by putting them from the climate chamber directly into the autosampler for immediate analysis. Unfortunately, frequent opening of the door of the climate cupboard resulted in unstable temperatures and error messages of the cupboard's control unit.

\section{Quantification}

Quantification was performed against a calibration line consisting of hexanal spiked into purified, hexanal-free sunflower oil at 6 levels ranging from 24 to $395 \mathrm{ppm}$. The purified oil was prepared by steam stripping $10 \mathrm{~kg}$ of sunflower oil in a pilot plant steam stripper for $3 \mathrm{~h}$ with $0.1 \mathrm{~kg}$ steam $/ \mathrm{h}$ at $220^{\circ} \mathrm{C}$ and $3 \mathrm{mbar}$. The hexanal level in the purified oil was below the detection limit of the GC-MS SIM method here developed. Because the automated Combipal agitator could not easily be operated at room temperature, the calibration standards were equilibrated 
(2 min) and measured at $30^{\circ} \mathrm{C}$. Since the large bottles containing the aged samples could not be put in the agitator oven, these were measured at $21^{\circ} \mathrm{C}$. Peak areas were converted to concentrations using the calibration line applying a correction factor to correct for the temperature difference between samples and calibration standards. This correction factor was calculated from the slope of a calibration line recorded at $30^{\circ} \mathrm{C}$ versus one recorded at $21^{\circ} \mathrm{C}$. The slope ratio was 1.53 .

\section{Spices and Herbs Studied}

A wide range of spices and herbs were included in the current study, these include Pepper (Piper nigrum L.), Sage (Salvia triloba L.), Clove (Syzygium aromaticum L.), Rosemary (Rosmarinus officinalis), and Ginger (Zingiber officinale Roscoe), with different levels of grinding and different origins. The spices and herbs were obtained from different local supermarkets and were either used as received, ground before use, or extracted with sunflower oil after which the extractant oil was used as the antioxidant mixture to test. No other treatment was applied.

\section{Results and Discussion}

\section{Threshold Determination}

Monitoring the production of newly formed oxidation species in systems with and without an antioxidant over time is a widely used method to assess anti-oxidant efficacy (8). A quantitative expression of the quality of an antioxidant mixture can be obtained from how long it takes to reach a certain threshold level of hexanal in these two systems. The ratio of the two times is the deceleration factor. The higher the deceleration factor, the more effective an antioxidant system is. The exact threshold level for hexanal used to determine the time at which the sample goes off is not extremely critical. Lipid oxidation usually proceeds exponentially after an initial lag phase. As long as the threshold level for hexanal is selected above the level in the lag phase, due to the steepness of the curve, the exact threshold value will only modestly affect the off-time. In our experiments we determined the threshold value by preparing standards of hexanal in sunflower oil and established organoleptically at what level the oil started to smell rancid. When sniffed at room temperature, this point was reached at $150 \mathrm{ppm}$ hexanal. To allow accurate quantification at this level, the quantification limits of the method should be lower than approximately 10 to $15 \mathrm{ppm}$.

\section{Fast GC-MS Analysis}

As outlined in the Introduction, due to the large number of spices and herbs with antioxidant effects and the possibility of interactions between them, very large numbers of hexanal measurements have to be done to find the optimum antioxidant mix. Fast GC-MS analysis is hence needed. The use of short columns with reduced inner diameters is a generic route towards faster GC (9). Here, a $150 \mu \mathrm{m}$ inner diameter column was selected as a compromise between speed and practical issues. Columns with diameters below this have poorer concentration detection limits due to the very limited maximum injection volume (10). Moreover, they have a limited working range, i.e., the range between detection limit and concentration at which stationary phase overloading occurs (11). The optimum carrier gas velocity was calculated using an Excel-based program (12). For the $6 \mathrm{~m}, 150 \mu \mathrm{m}$ column used here it was approximately $65 \mathrm{~cm} / \mathrm{s}$. The column was operated at 1.5 times this velocity (approximately $95 \mathrm{~cm} / \mathrm{s}$ ), giving a dead time of approximately $6 \mathrm{~s}$. At this velocity the separation speed, i.e., the number of plates generated per time unit, is at its maximum (13). A temperature programming rate of $80^{\circ} \mathrm{C} / \mathrm{min}$ was calculated to be the optimal (14). Injection volume and split ratio were optimized to minimize the injection contribution to peak width while meeting the required concentration detection limits. A representative example chromatogram of a heavily oxidized oil is shown in Figure 1.

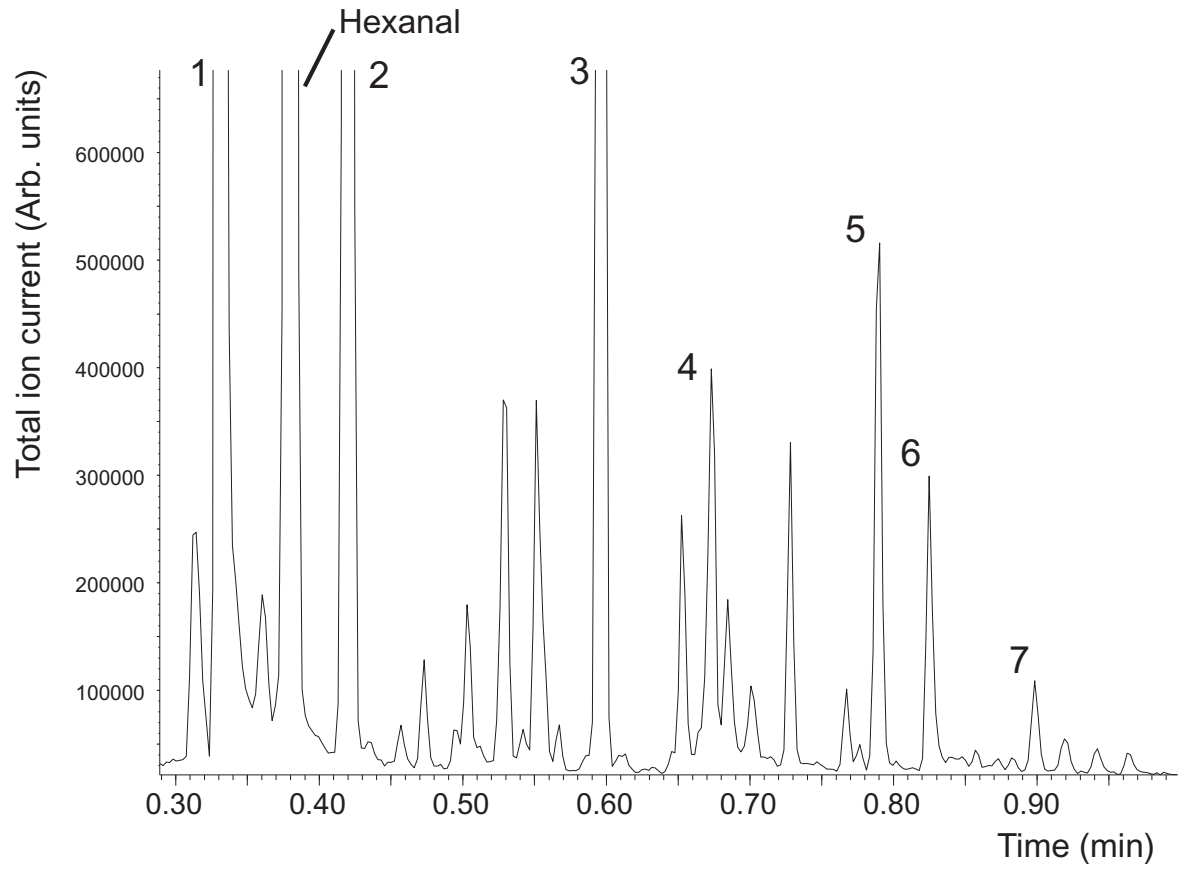

Figure 1. Fast GC-MS analysis of the headspace of a heavily oxidized sunflower oil (TIC). Peak identification ( $t=$ tentative). $1=1$-Pentanol, $2=$ Formic acid, pentyl ester, $3=$ Pentane, 1-iodo (t), $4=$ Hexanoic acid, $5=$ Eucalyptol, $6=2$-Octenal, 7 = Nonanal. 
The hexanal level in this sample is around $350 \mathrm{ppm}$. Although hexanal elutes within $0.4 \mathrm{~min}$, the presence of higher boiling compounds requires a subsequent column bake out step. To minimize the time for reconditioning, the programming rate was increased to the maximum achievable value of $100^{\circ} \mathrm{C} / \mathrm{min}$. The chromatogram in Figure 1 shows the typical compounds expected for oxidized sunflower oil with hexanal, pentanol, and 2-octenal as important oxidation products. In TIC mode the quantification limit was approximately $5 \mathrm{ppm}$. To allow measurement of also the lowest concentrations of hexanal in the starting samples, the system was operated in the SIM mode. Three ions were monitored, $\mathrm{m} / \mathrm{z} 56$ was selected as the quantifier ion, and $m / z 72$ and 82 as qualifier ions. Ion ratios $m / z$ 72 vs 56 and $\mathrm{m} / \mathrm{z} 82$ vs 56 were 0.31 (RSD 1.6\%) and 0.26 (RSD 6.8\%), respectively, at the lowest level of the calibration line (24 ppm). The detection limit in SIM mode, determined from the concentration that gave a peak with a signal to noise ratio of 4 for ion $\mathrm{m} / \mathrm{z} 56$, was better than $0.2 \mathrm{ppm}$. The recovery of the method was established by adding a standard solution of hexanal in stripped sunflower oil onto the salt matrix with quantification against the calibration line prepared using hexanal in stripped sunflower oil. The recovery value established was $104 \%$. The repeatability of the GC-MS hexanal head-space measurement was tested by analyzing a sample with a value of around $100 \mathrm{ppm} 4$ times. The repeatability was better than $4 \%$. Note that this is the repeatability of the GC-MS measurement only. The repeatability of the actual aging reaction is assessed below.

\section{Accelerated Sample Aging}

The fast GC-MS measurement of hexanal provides a rapid readout for the aging of sunflower oil. Next, a realistic model system should be developed for the accelerated aging process. This should be as similar as possible to the food system in which the antioxidants will be applied, yet fast enough to obtain a measurable degree of oxidation in a reasonable time frame. The food systems of interest here were dry powdered products, in particular dry soups, sauces and meal starters. Such products consist of dried vegetables or other dried pieces like dried meat pieces or croutons, various spices and herbs, and salt. A vegetable oil like sunflower oil is generally added to such products for dust control in the factory environment or during the preparation in the kitchen, as well as for optimized flavor experience. The high level of salt results in a very low water activity and a large surface area, making the food product very amenable to lipid oxidation (15). In our model system this was mimicked by using a test sample consisting of fine salt to which the sunflower oil and antioxidant spices and herb mixtures were added. To enhance oxidation rates and allow to study oxidation at normal storage temperatures, a low volume of oil was spread out as a very thin film on a large quantity of salt. Figure 2 shows the analysis of a control sample without spices and herbs and two samples of different spices mixes. The horizontal line gives the threshold value of $150 \mathrm{ppm}$. For the reference sample without spices the time to exceed the limit value is approximately $40 \mathrm{~h}$, for the two different spices mixes it is approximately $160 \mathrm{~h}$ and $300 \mathrm{~h}$. This results in deceleration factors of 4.0 and 7.6, respectively.

\section{Method Repeatability}

To assess the repeatability of deceleration factors, a series of aging experiments of 18 different spices and herbs mixtures was performed with all samples being studied as fully independent triplicates. To eliminate errors due to inhomogeneities in the spices and herbs, larger amounts of these, typically $1 \mathrm{~g}$, were extracted with $10 \mathrm{~mL}$ of sunflower oil in $20 \mathrm{~mL}$ head-space vials for $4 \mathrm{~h}$ using a test tube rotator (model 34528, Snijders Scientific, Tilburg, the Netherlands) operated at $15 \mathrm{rpm}$. After extraction, $1 \mathrm{~g}$ of the extractant oil was added to $100 \mathrm{~g}$ of salt, the sample was mixed by shaking (see above) and aging was performed at $30^{\circ} \mathrm{C}$. The results of these experiments, expressed as the deceleration factors, are summarized in Figure 3. RSD values for the experiments are typically better than $10 \%(n=3)$, where the repeatabilities were generally poorer for the mixes with the higher deceleration factors. This indicates that the oxidation process itself is rather repeatable, but if a spices and herbs mix interferences with this oxidation process the exact magnitude depends on other factors that are apparently not perfectly controllable.

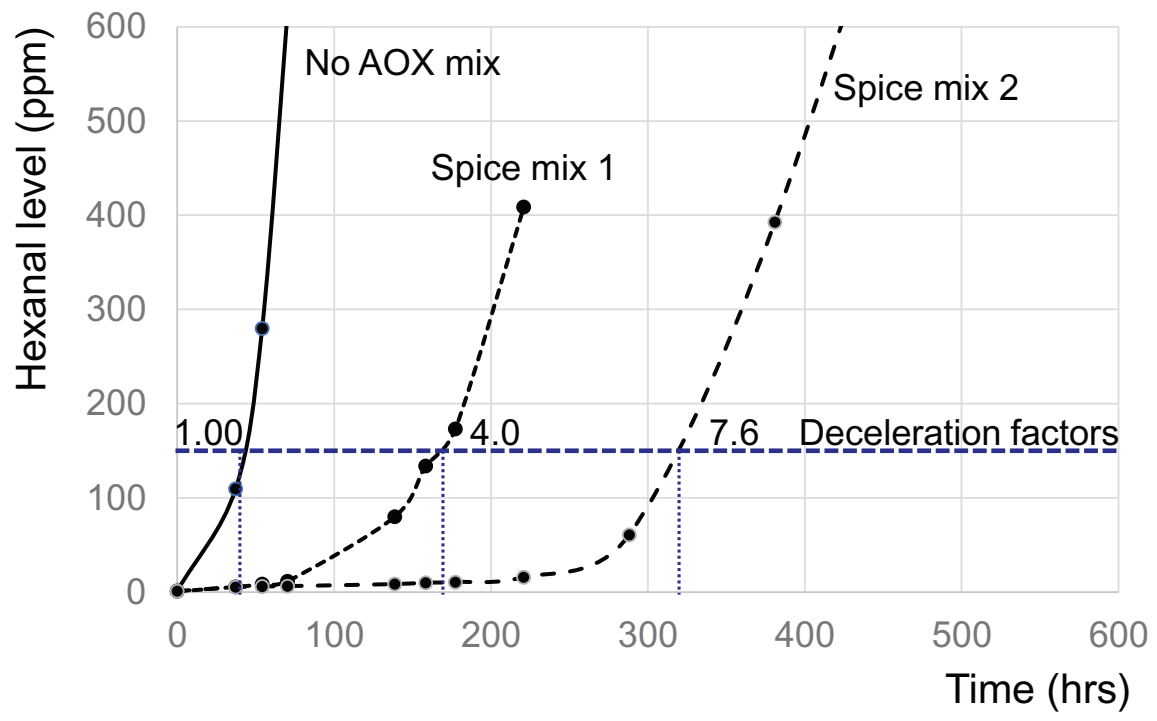

Figure 2. Representative hexanal formation curves for two spice mixes and the reference sample with no natural antioxidant (AOX). 


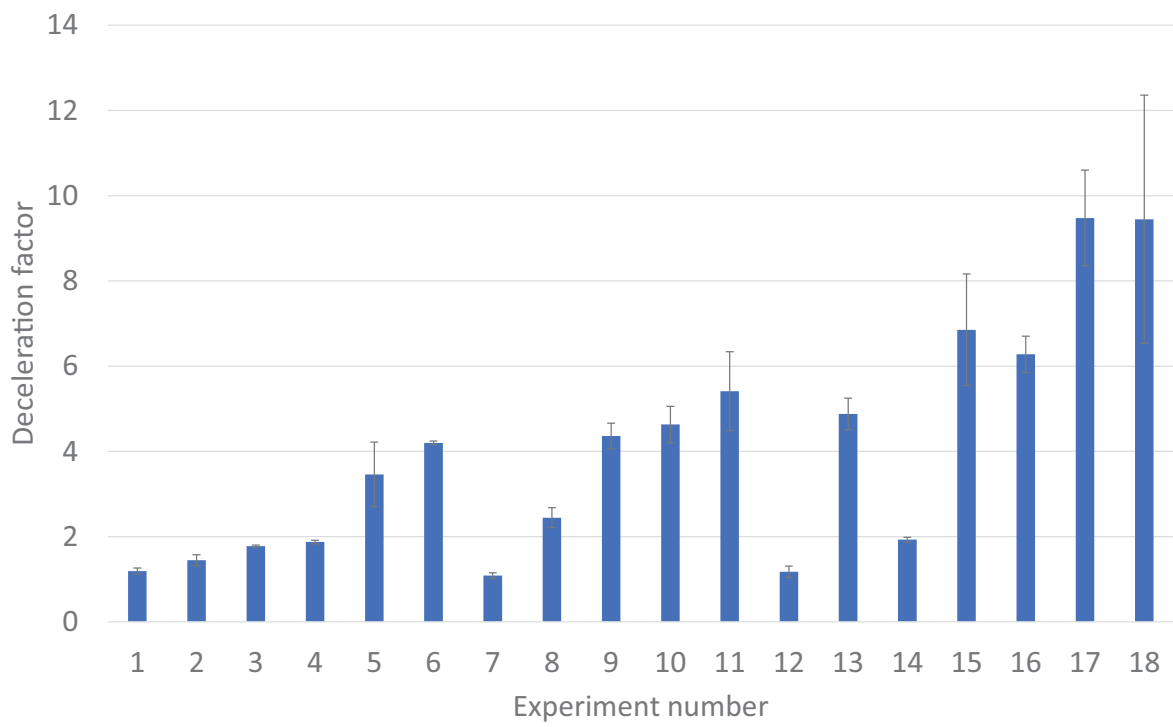

Figure 3. Deceleration factors for a selected set of experiments. Error bars indicate plus or minus twice the standard deviations $(n=3)$. Different experiment numbers indicate different spices and herbs mixtures.

Table 1. Deceleration factors of pure ingredients at different levels. Weight percentages are calculated vs the total mass of salt (100 g) plus sunflower oil (1 g)

\begin{tabular}{lcccr}
\hline Ginger powder, wt-\% & Rosemary powder, wt-\% & Sage powder, wt-\% & Ratio G:R:S & Deceleration factor (DF) \\
\hline 0.017 & - & - & $1: 0: 0$ & 2.3 \\
0.033 & - & - & $2: 0: 0$ & 3.9 \\
0.067 & - & - & $4: 0: 0$ & 13.7 \\
- & 0.017 & - & $0: 1: 0$ & 2.2 \\
- & 0.033 & - & $0: 2: 0$ & 4.6 \\
- & 0.050 & 0.017 & $0: 3: 0$ & 10.1 \\
- & - & 0.033 & $0: 0: 1$ & 3.6 \\
- & - & 0.050 & $0: 0: 2$ & 7.5 \\
\hline
\end{tabular}

All results reported are the average of three independent measurements.

\section{Antioxidant Efficacy and Synergy Effects}

To test the efficacy of a combination of spices and herbs that had previously been identified as synergistic (16), deceleration factors were measured at different levels. Ginger, rosemary, and sage powder (size range approximately 0.2 to $0.5 \mathrm{~mm}$ ) were included in the study. Table 1 gives a summary of the results. A significant delay in oxidation is seen for all three samples studied, even at levels as low as $0.017 \%$. At all concentration levels tested sage is the strongest antioxidant. Rosemary is more effective than ginger at intermediate level, whereas ginger is slightly better than rosemary at low levels.

Synergy effects between different spices and herbs were studied by comparing the deceleration factors obtained when two or more ingredients were combined with the sum of the deceleration factors of the individual species. A small selection of the data is presented in Table 2. In all cases, a significant synergy effect was seen. As an example, in the first experiment (Exp. No. 1), the combination of $0.067 \mathrm{wt}$ \% ginger, $0.017 \mathrm{wt}-\%$ rosemary, and $0.017 \mathrm{wt}$ \% sage resulted in a deceleration factor of 63.4. The individual deceleration factors for these three ingredients at the same concentrations were 13.7, 2.2, and 3.6, respectively. The summed deceleration factor of the individual ingredients is 19.5 , which is much lower than the experimental deceleration factor for the mixed ingredients of 63.4. Several of the ginger/rosemary/sage mixes tested resulted in such a strong retardation of lipid oxidation that the hexanal level was still below the $150 \mathrm{ppm}$ threshold value at the end of the 11 weeks measurement period. Extrapolation of the hexanal curves resulted in deceleration factors as high as 160 to 180 . The values reported in Table 2, however, were calculated using the most conservative approach that the off-time equaled the last measurement point (77 days). For these samples the deceleration factors and synergy effects reported are the minimum values and the actual values will even be higher. A representative SIM chromatogram of the analyses is shown in Figure 4.

Since the exact curve of the hexanal formation cannot be predicted beforehand, hexanal measurements should be performed at a rather high frequency, initially typically once every day, reduced to once a week for samples that are highly stable. This results in large numbers of samples to be tested. In our experiments we typically performed the study with sample sets of 80 to 100 samples which have to be measured on one day. This results in a total net measurement time of 4 to $5 \mathrm{~h}$, leaving enough time for recording calibration lines, peak integration, reporting of the data, and system maintenance. Clearly, this massive number of samples could only be processed thanks to 
6 | Janssen et al.: Journal of AOAC INTERNATIONAL Vol. 0, No. 0,

Table 2. Deceleration factors and synergy effects of mixed spices and herbs powders

\begin{tabular}{|c|c|c|c|c|c|c|}
\hline Exp. No. & $\begin{array}{c}\text { Ginger } \\
\text { powder, wt-\% }\end{array}$ & $\begin{array}{c}\text { Rosemary } \\
\text { powder, wt-\% }\end{array}$ & $\begin{array}{c}\text { Sage } \\
\text { powder, wt-\% }\end{array}$ & $\begin{array}{l}\text { Ratio } \\
\text { G: R: S }\end{array}$ & $\begin{array}{c}\text { Deceleration } \\
\text { factor }\end{array}$ & $\begin{array}{c}\text { Synergy } \\
\text { effect }\end{array}$ \\
\hline 1 & 0.067 & 0.017 & 0.017 & 4:1:1 & 63.4 & 44.0 \\
\hline 2 & 0.017 & 0.050 & 0.017 & 1:3:1 & 35.5 & 20.0 \\
\hline 3 & 0.067 & 0.050 & 0.017 & $4: 3: 1$ & $>117.3^{a}$ & $>90.0^{\mathrm{a}}$ \\
\hline 4 & 0.033 & 0.033 & 0.033 & $2: 2: 2$ & 56.4 & 40.4 \\
\hline 5 & 0.017 & 0.017 & 0.050 & $1: 1: 2$ & 63.4 & 41.7 \\
\hline 6 & 0.067 & 0.017 & 0.050 & $4: 1: 3$ & $>117.3^{a}$ & $>84.2^{\mathrm{a}}$ \\
\hline 7 & 0.017 & 0.050 & 0.050 & $1: 3: 3$ & $>113.3^{b}$ & $>83.6^{\mathrm{b}}$ \\
\hline 8 & 0.067 & 0.050 & 0.050 & $4: 3: 3$ & $>117.3^{\mathrm{a}}$ & $>76.3^{\mathrm{a}}$ \\
\hline
\end{tabular}

${ }^{a}$ Minimum value, all triplicate specimens still stable at end of stability test.

${ }^{\mathrm{b}}$ Minimum value, one specimen of triplicate still stable at end of stability test.

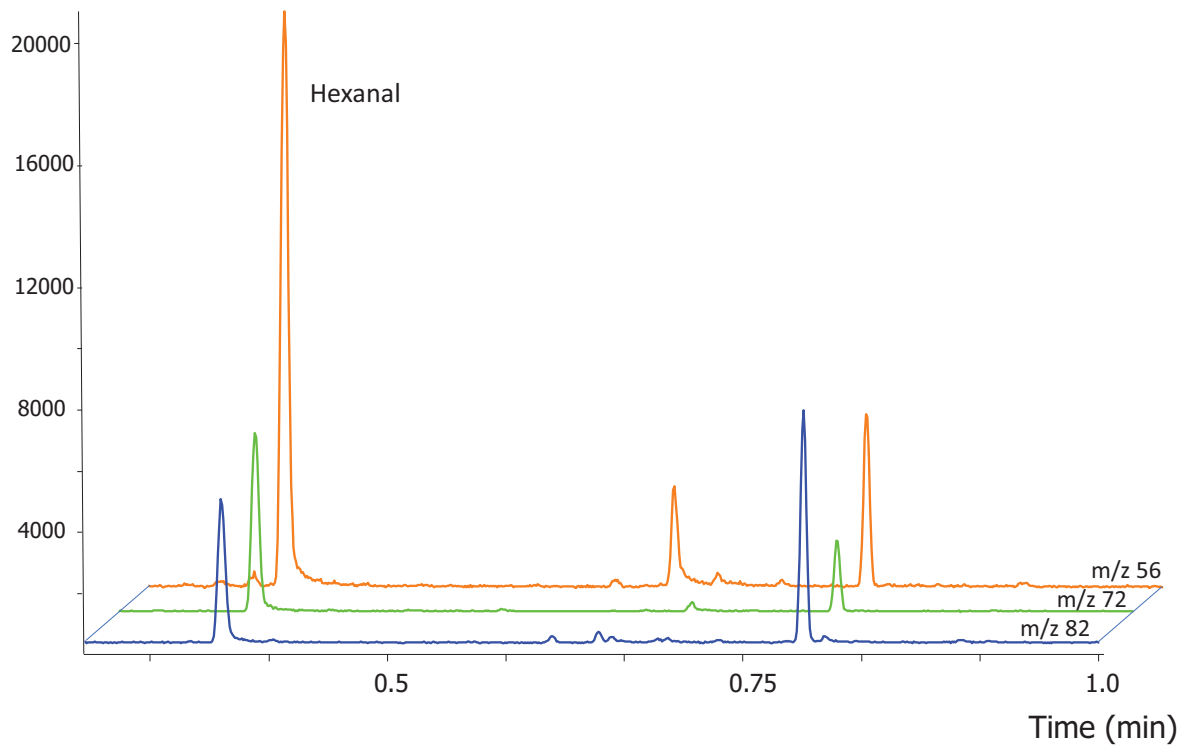

Figure 4. Representative fast GC-MS SIM analysis of a sample from the aging experiment (hexanal level 14 ppm). Time and intensity scales are shifted for clarity.

the use of the fast GC method developed here. In practical use the method turned out to be highly reliable. Because only clean gaseous head-space samples are injected the system is very stable. Currently more than 5000 injections have been performed on the same column with no cleaning of the MS ion source. The only maintenance needed was the frequent replacement of the injector septum. Further automation of the method was considered, but not further pursued. The size of the flasks in which aging was performed would require impractically large autosampler racks or trays.

Evidently all spices and herbs tested can have a contribution to the flavor and taste of a product, so selection of the appropriate mixture will also depend on the food product in which it will be applied. With the fast method developed here, productmatched effective natural antioxidant mixes can be rapidly evaluated for their lipid oxidation inhibitory potential.

\section{Conclusions}

Spices and herbs are very efficient natural inhibitors of lipid oxidation. Monitoring the delay of hexanal formation is an efficient manner to quantify the efficacy of specific mixtures of them. Because of the many materials available and the possibility of mutual interactions, large sample numbers have to be screened. Using fast GC-MS on a short $6 \mathrm{~m}, 150 \mu \mathrm{m}$ internal diameter column, hexanal can be eluted in less than approximately $20 \mathrm{~s}$ with a cycle time of around $2 \mathrm{~min}$. The total time required for the analysis of one sample including calibration, integration, reporting, and system maintenance is around $5 \mathrm{~min}$. Standard GC-MS equipment can be used, but to achieve the high temperature programming rate required an oven insert pillow should be installed. Huge numbers of samples can be analyzed without problem, allowing for the experimental evaluation of large numbers of mixtures. With the right mixture of spices and herbs, deceleration factors of more than 100 can be reached.

Conflict of Interest: The authors are employed by Unilever, a multinational consumer goods company.

\section{References}

1. Embuscado, M.E. (2015) J. Funct. Foods 18, 811-819. doi: 10.1016/j.jff.2015.03.005

2. Yanishlieva, N.V., Marinova, E., \& Pokorný, J. (2006) Eur. J. Lipid Sci. Technol. 108, 776-793. doi:10.1002/ejlt.200600127

3. Shahidi, R. \& Zhong, Y. (201) Chem. Soc. Rev. 39, 4067-4079. doi:10.1039/B922183M 
4. Becker, E.M., Nissen, L.R., \& Skibsted, L.H. (2004) Eur. Food Res. Technol. 219, 561-571. doi:10.1007/s00217-004-1012-4

5. Azarbad, M.H., \& Jeleń, H. (2015) Food Anal. Methods 8, 1727-1733. doi:10.1007/s12161-014-0043-0

6. Apak, R., Özyürek, M., Güçlü, H., \& Çapanoğlu, E. (2016) J. Agric. Food Chem. 64, 997-1027. doi:10.1021/acs.jafc.5b04739

7. Masuda, T., Inaba, Y., Maekawa, T., Takeda, Y., Tamura, H., \& Yamaguchi, H. (2002) J. Agric. Food Chem. 50, 5863-5869. doi: 10.1021/jf025605o

8. Laguerre, M., Lecomte, J., \& Villeneuve, P. (2007) Prog. Lipid Res. 46, 244-282. doi:10.1016/j.plipres.2007.05.002

9. Korytár, P., Janssen, H.-G., Matisová, E., \& Brinkman, U.A.T. (2002) Trends Anal. Chem. 21, 558-572. doi:10.1016/S01659936(02)00811-7
10. Noy, T., Curvers, J., \& Cramers, C.A. (1986) J. High Resol. Chromatogr. 9, 752-759. doi:10.1002/jhrc.1240091208

11. Dömötörová, M., Kirchner, M., Matisová, E., \& de Zeeuw, J. (2006) J. Sep. Sci. 29, 1051-1063. doi:10.1002/jssc.200500472

12. Beens, J., Janssen, H.-G., Adahchour, M., \& Brinkman, U.A.T. (2005) J. Chromatogr. A 1086, 141-150. doi:10.1016/j.chroma. 2005.05.086

13. Blumberg, L.M. (1997) J. High Resol. Chromatogr. 20, 679-687. doi:10.1002/jhrc.1240201212

14. Blumberg, L.M., \& Klee, M.S. (1998) Anal. Chem. 70, 3828-3839. doi:10.1021/AC971141V

15. Labuza, T.P. (1980) Food Technol. 34, 36-41

16. Gah, C.I., Jonas, V., \& Spraul, M.H. (2019) Antioxidant composition. International Patent Application No. WO 2019/170737 A1 